\title{
Primary Extra-renal Wilms' Tumor in Urinary Bladder: Rare Presentation of a Common Pediatric Malignancy
}

\author{
Irfana Ishaq Sindhu, Haleema Saeed, Rabia Wali and Asma Mehreen
}

\begin{abstract}
In pediatric population, the most common solid malignant tumor of the kidney is Wilms' tumor (WT). Incidence of ExtraRenal Wilms' Tumor (ERWT) is immensely unusual. Prognosis of ERWT is comparable to renal WT. Hence, diagnosis, staging workup and start of treatment in a timely manner is very essential. The most common location of ERWT is the retroperitoneum. The staging of ERWT is done according to National Wilms' Tumor Study group (NWTS). ERWT is considered as stage II or higher; because it is outside renal capsule. A 6-year boy presented with complaints of increased frequency of micturition and difficulty in urination for 15 months. Biopsy of bladder mass showed WT by immunohistochemistry stains such as WTI and Desmin. Cross-sectional imaging showed both kidneys clear of any tumor. Hence, the final diagnosis of ERWT was made.
\end{abstract}

Key Words: Extra renal Wilms' tumor, National Wilms tumor study, Teratoma, Chemotherapy.

\section{INTRODUCTION}

Wilms' tumor (WT) accounts for more than two-thirds of pediatric renal masses with most of the children presenting in the first five years of age. Extra-renal Wilms tumor (ERWT) is a rare malignancy, which was first described by Moyson et al. in 1961.1 The incidence of ERWT is about $0.5-1 \%$ of all cases of WT. Previously, about 200 cases of ERWT have been reported in literature, including those in adults. ${ }^{2}$

WT may present outside the kidney, in the form of metastatic disease and WT arising in a teratoma, apart from the primary ERWT. It is imperative to examine both kidneys for primary disease intraoperatively and for teratoid component, to examine the whole specimen of the resected tumor. Surgical resection followed by adjuvant chemotherapy is the treatment of choice for all stages and histology types of ERWT. Radiotherapy is given to those patients in whom the tumor is unresectable or have gross residual disease, recurrence or metastasis. ${ }^{3-5}$

The overall survival outcome at 2 years in the reported ERWT cases is approximately $85 \%$ and mortality rate $5 \%$, which is equal to that of renal WT. We present a successfully treated case of ERWT occurring in urinary bladder, coupled with review of relevant literature.

Department of Pediatric Oncology, Shaukat Khanum Memorial Cancer Hospital and Research Centre, Lahore, Pakistan

Correspondence: Dr. Irfana Ishaq Sindhu, Department of Pediatric Oncology, Shaukat Khanum Memorial Cancer

Hospital and Research Centre, Lahore, Pakistan

E-mail: irfanaishaq@skm.org.pk

Received: August 10, 2018; Accepted: January 03, 2019

\section{CASE REPORT}

A six-year boy presented with increased frequency of micturition and difficulty in urination for past 15 months. Ultrasound kidney ureter bladder (KUB) showed a polyp in right lateral bladder wall. Open polypectomy was done in October 2015. There was suspicion of malignancy but the child was lost to follow-up. He remained asymptomatic for the next one year. Then, again, he developed complaints of dysuria and frequent micturition. Ultrasound KUB showed mild generalised wall thickening of urinary bladder along with a pedunculated mass measuring about $23 \times 22 \mathrm{~mm}$, attached to bladder base near prostatic urethral opening, projecting into the lumen and showed blood flow on color Doppler. Repeated polypectomy was done in August 2016. Histopathology showed a malignant round blue cell tumor composed predominantly of immature tubules with associated spindle cell (mesenchymal) stroma. No heterologous differentiation noted (Figure 1). WT1 and desmin were positive. Predominant biphasic morphology of the tumor, small round (balstemal) and immature tubular pattern confirmed the diagnosis of ERWT. Cross-sectional imaging of pelvis showed urinary bladder to be well distended without obvious nodular growth or wall thickening (Figure 2). No dilatation of both ureters and no significant pelvic or inguinal lymph adenopathy were seen. Visualised bowel also appeared unremarkable. There was no ascites. Marrow signals appeared unremarkable. Tumor was classified as stage III with favourable histology by using SIOP Protocol and treated according to SIOP WT guidelines. Actinomycin, vincristine, and doxorubicin chemotherapy for 28 weeks was given along with radiation of $15.5 \mathrm{~Gy}$ to bladder. End of treatment (EOT) cross imaging showed well distended, urinary bladder without definite mass lesion or wall thickening. No significant pelvic or inguinal 


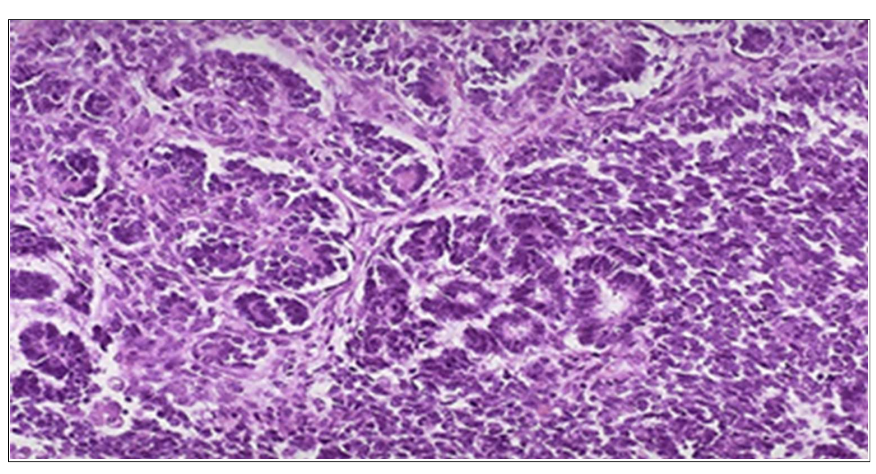

Figure 1: Histopathology showed predominantly biphasic Wilm's tumor composed of immature tubules and small rounds cells. Small areas of mesenchymel component are also seen.

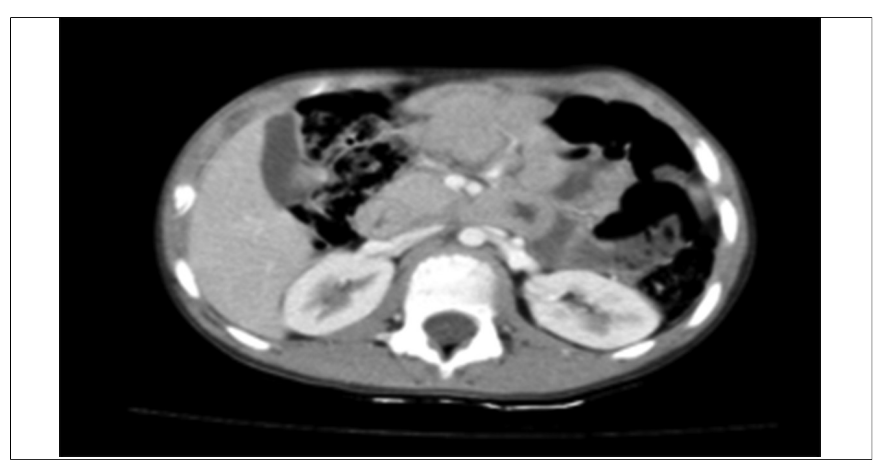

Figure 2: CT abdomen showed bilateral normal kidneys.

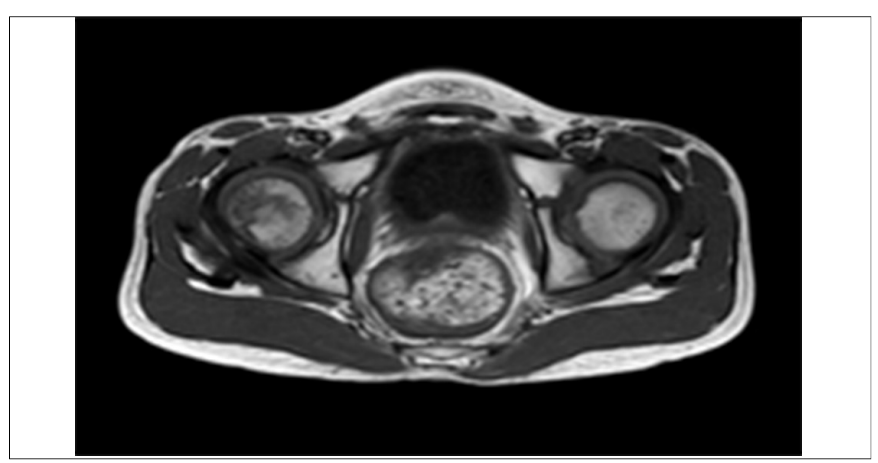

Figure 3: MRI pelvis shows no residual disease in bladder.

lymphadenopathy was seen. Both kidneys were tumorfree (Figure 3). Distal ureters were not dilated. Now, he is on long-term follow-up with ultrasound KUB and X-ray chest.

\section{DISCUSSION}

In pediatric age group, the most common malignant tumor arising from kidneys is the WT with rare reports of ERWT. Common sites of ERWT are retroperitoneum, inguinal canal, lumbosacral region, pelvis, mediastinum, chest wall, female genital tract, spermatic cord and the para-testicular region. 6-11 Majority of ERWT are located in the retroperitoneum. In this case, ERWT was present in the urinary bladder, a rare site for this tumor.

ERWT in urinary bladder, to the authors' best knowledge, is the first case to be reported. There was a case report in which WT was in right renal pelvis with extension into the bladder reported by Nagahara et al. ${ }^{7}$

The age of presentation is between 2 months and 10 years, while in renal WT is 44 months. A 77-year female was diagnosed with uterine ERWT, she was the oldest reported patient. It is more prevalent in females $(56.6 \%)$ than in males (43.4\%).

The most common genetic mutation is the WTI gene mutation, found in $25 \%$ cases of ERWT. ERWT originates from ectopic mesonephric blastema or primitive mesodermal tissue, as suggested by Connheim's rest theory. ${ }^{3}$

ERWT may be found in two other situations: metastatic disease and nephroblastoma emerging in a teratoma, exclusive of primary ERWT. It is essential in cases of ERWT to assess the kidneys for primary tumor preoperatively and to research for teratoid component in specimens postoperatively. ${ }^{8}$ There are about 54 reported cases of teratoma with nephroblastoma. Most common location is the kidney.

ERWT has no specific clinical characteristics, and clinical symptoms depend on location and stage of tumor. ERWT is usually diagnosed after resection and pathologic evaluation of the specimen. ERWT should have three features for its diagnosis according to national Wilms tumor study (NWTS). First, tumor should not be present in both kidneys. Second, on histology triphasic pattern should be present, and third, on histology, there should be no teratoma or renal cell carcinoma. ${ }^{9}$ This case fulfilled these criteria for the diagnosis of ERWT.

In previous reported cases, occurrence of ERWT in a horseshoe kidney was almost 7\%.10 Dysraphism is the second common abnormality associated with ERWT. These congenital malformations were not present in this patient.

SIOP protocol is used for the management of WT in kidneys, except when there is suspicion about diagnosis preoperatively, as in the case presented. The SIOP protocol reduces the risk of tumor rupture. It is appropriate for us because most patients in the setup present with advanced disease.

According to histology, WT is divided into low-risk, intermediate-risk, and high-risk. By this stratification, intensity of treatment is reduced for low risk patients in SIOP protocol. Nowadays, treatment of ERWT is similar to renal WT. In this case, the patient first underwent surgery then given neoadjuvant chemotherapy with radiotherapy.

The percentage of local relapse in ERWT is reported as $11 \%$ in favourable histology; while in renal WT, it is $15 \%$. Overall survival rates of stage II, III and IV ERWT are $70 \%, 23 \%$ and $6 \%$, respectively. Two years event-free survival of ERWT was $85 \%$ and death rate $5 \%$, which is 
similar to renal WT. Radiotherapy is reserved for unresectable, residual and metastatic disease.

ERWT is extremely rare. It is important to keep in differential diagnosis in any child presenting with urinary symtoms and polypoidal lesions in the bladder to avoid delay in initiating treatment.

\section{REFERENCES}

1. Moyson F, Maurus-Desmarez R, Gompel C. Mediastinal Wilms' tumor? Acta Chir Belg 1961; Suppl 2:118-28.

2. Taguchi S, Shono T, Mori D, Horie H. Extrarenal Wilms tumor in children with unfavorable histology: A case report. $J$ Pediatr Surg 2010; 45:e19-22.

3. Shojaeian R, Hiradfar M, Sharifabad PS, Zabolinejad N. Extrarenal Wilms' tumor: Challenges in diagnosis, embryology, treatment and prognosis. Wilms Tumor 2016.

4. Song JS, Kim IK, Kim YM, Khang SK, Kim KR, Lee Y. Extrarenal teratoid Wilms' tumor: Two cases in unusual locations, one associated with elevated serum AFP. Pathol Int 2010; 60:35-41.
5. Apoznanski W, Sawicz-Birkowska K, Palczewski M, Szydelko T. Extrarenal nephroblastoma. Cent European J Urol 2015; 68: 153-6.

6. Sastri J, Dedhia R, Laskar S, Shet T, Kurkure P, Muckaden M. Extra-renal Wilms' tumour: Is it different? Pediatr Nephro 2006; 21:591-6.

7. Nagahara A, Kawagoe M, Matsumoto F, Tohda A, Shimada K, Yasui $\mathrm{M}$, et al. Botryoid Wilms' tumor of the renal pelvis extending into the bladder. Urology 2006; 67:845-e15.

8. Li Y, Lei C, Xiang B, Li F, Wang C, Wang Q, et al. Extrarenal teratoma with nephroblastoma in the retroperitoneum: Case report and literature review. Medicine (Baltimore) 2017; 96: e8670.

9. Thakkar NC, Sarin YK. Extra-renal Wilms' tumor: A rare diagnosis. APSP J Case Rep 2015; 6:17.

10. Baskaran D. Extrarenal teratoid Wilms' tumor in association with horseshoe kidney. Indian J Surg 2013; 75:128-32.

11. Khalid A, Faizan M, Khan S, Najamuddin, Talat N, Rathore Z. Extra-renal Wilm's tumor in a 3 years old boy: A case report. J Cancer Prev Curr Res 2017; 7:00254. 